\title{
Dermoscopy in General Dermatology (Non-Neoplastic Dermatoses): The Journey So Far
}

Enzo Errichetti

Received: July 29, 2021 / Accepted: October 8, 2021 / Published online: October 19, 2021

(C) The Author(s) 2021

Keywords: Dermoscopy; Entomodermoscopy; Inflammoscopy; Pigmentaroscopy

\section{COMMENTARY}

Although classically used for assisting the diagnosis of skin neoplasias, dermoscopy is increasingly gaining significant appreciation also in the field of general dermatology, including inflammatory, pigmentary, infectious, and infiltrative dermatoses [1]. Many articles on this topic have been published over the last few years, addressing common but also less frequent skin conditions whose recognition may be facilitated by dermoscopic examination [1]. Notably, several literature reviews have tried to summarize the possible indications of dermoscopy when it comes to the main non-neoplastic dermatoses, yet there is little awareness on the use of such a technique for rarer diseases [2-6]. To deal with such a gap, an in-depth search of the literature was performed to give an up-to-date temporal overview on all the nonneoplastic dermatoses whose dermoscopic features have been described, thus increasing the

E. Errichetti $(\bowtie)$

Institute of Dermatology, "Santa Maria della Misericordia" University Hospital, Piazzale Santa Maria della Misericordia, 15, 33100 Udine, Italy e-mail: enzoerri@yahoo.it physicians' awareness on the possible diagnostic uses of dermoscopy in general dermatology. In detail, a search of the articles published up to 2020 was performed through the PubMed electronic database using the search terms "dermoscopy", "dermatoscopy", "videodermatoscopy", or "epiluminescence microscopy". Abstracts and titles were screened to identify papers describing dermoscopic findings of at least one inflammatory, pigmentary, infiltrative, or infectious skin disease (hair, nail, and mucosal conditions were not considered). A total of 6710 publications were analyzed and 305 different dermatoses (also including relevant disease variants characterized by dermoscopic peculiarities) with at least one dermoscopic description were identified. Figure 1 depicts the temporal distribution of the first dermoscopic report of such dermatoses, while Table 1 shows a complete list of the conditions according to the year of the first description.

This literature search highlights that the potential diagnostic applications of dermoscopy in non-neoplastic dermatoses are quite numerous. The initial reports in this field date back to 1997, when dermoscopic features of scabies and larva migrans were first described $[7,8]$, while it was not until 2001 that findings of non-infectious inflammatory dermatoses start to be reported. Indeed, besides tinea nigra and nodular scabies, dermoscopic descriptions of 


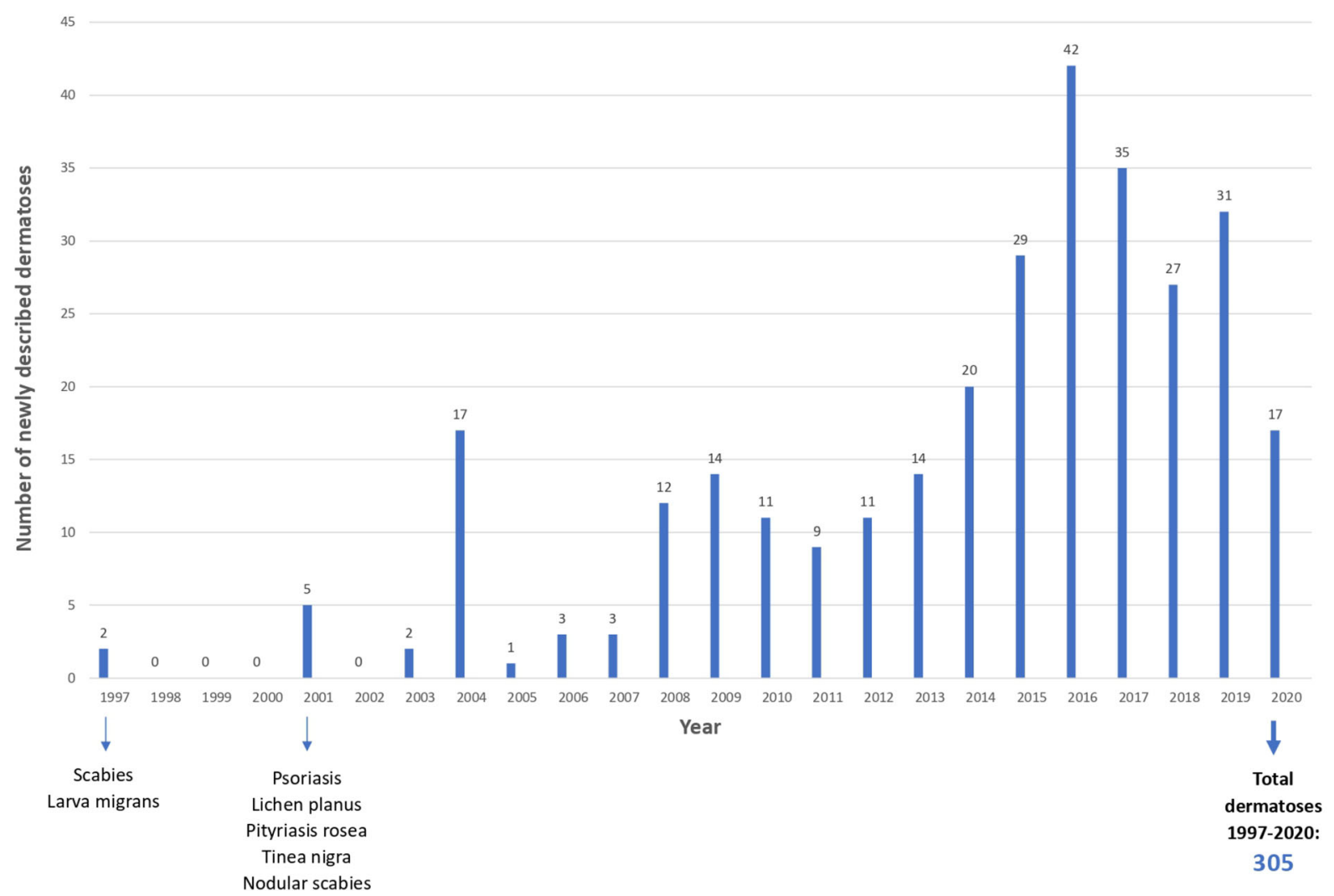

Fig. 1 Temporal distribution of the number of dermatoses whose dermoscopic features were described for the first time; dermoscopy of scabies and larva migrans were the first non-neoplastic dermatoses to be described (in 1997),

psoriasis, lichen planus, and pityriasis rosea were published during that year. Afterwards, apart from a leap in the number of new dermoscopic reports of non-neoplastic dermatoses in 2004 as a result of the publication of an article describing the vascular structures seen in several conditions [9], few dermatoses were investigated from a dermoscopic point of view till 2008. Then a gradual increase took place over the time with the peak being observed in 2016 , when dermoscopic features of a total of 42 new dermatoses were reported.

Importantly, more than $95 \%$ of the studies published on dermoscopy of non-neoplastic dermatoses have a low level of evidence (case reports/case series), with some of them revealing only poorly specific dermoscopic findings [1]. Nevertheless, if dermoscopic examination is used as the second step of a "two-step while psoriasis, lichen planus, and pityriasis rosea were the first non-infectious conditions for which a dermoscopic report was available (in 2001)

procedure", after narrowing the differential diagnosis to two or few conditions on clinical grounds, less specific features may still come in handy as long as they reflect relevant histological differences [2]. For example, purpuric spots are considered unspecific by themselves but their presence may help distinguish urticaria vasculitis from urticaria as they are seen only in the former as a result of erythrocytes extravasation [6].

In conclusion, diagnostic applications of dermoscopy in general dermatology have become quite broad over the time and clinicians should be aware of dermatoses that may benefit from this technique because, if used with a systematic approach, it may significantly improve diagnostic accuracy of both common and less frequent conditions. 
Table 1 List of non-neoplastic dermatoses whose dermoscopic features have been reported in the literature with the year of the first description

\begin{tabular}{|c|c|c|}
\hline Year & $\begin{array}{l}\text { Number of newly described } \\
\text { dermatoses }\end{array}$ & Dermatoses \\
\hline 1997 & 2 & Scabies, cutaneous larva migrans \\
\hline 1998 & 0 & - \\
\hline 1999 & 0 & - \\
\hline 2000 & 0 & - \\
\hline 2001 & 5 & Psoriasis, lichen planus, pityriasis rosea, tinea nigra, nodular scabies \\
\hline 2002 & 0 & - \\
\hline 2003 & 2 & Urticaria, urticaria vasculitis \\
\hline 2004 & 17 & $\begin{array}{l}\text { Disseminated superficial actinic porokeratosis, Sweet syndrome, tinea corporis, } \\
\text { tungiasis, vitiligo, plane warts, Darier disease, venous stasis dermatitis, erythema } \\
\text { annulare centrifugum, erythema multiforme, common folliculitis, urticaria } \\
\text { pigmentosa, molluscum contagiosum, necrobiosis lipoidica, panniculitis, perniosis, } \\
\text { lichen aureus }\end{array}$ \\
\hline 2005 & 1 & Cutaneous dystrophic calcifications \\
\hline 2006 & 3 & $\begin{array}{l}\text { Pseudofolliculitis barbae, spider leg spines skin reaction, epidermodysplasia } \\
\text { verruciformis }\end{array}$ \\
\hline 2007 & 3 & Xanthogranuloma, circumscribed acral hypokeratosis, furuncular myiasis \\
\hline 2008 & 12 & $\begin{array}{l}\text { Palmoplantar psoriasis, solitary cutaneous reticulohistiocytosis, scurvy, trichostasis } \\
\text { spinulosa, genital warts, verruca vulgaris, Degos disease, chronic hand eczema, } \\
\text { Dowling-Degos disease, exogenous ochronosis, lichen striatus, melasma }\end{array}$ \\
\hline 2009 & 14 & $\begin{array}{l}\text { Porokeratosis of Mibelli, genital psoriasis, Rosai-Dorfman disease, blue vitiligo, } \\
\text { plantar warts, cutaneous leishmaniasis, discoid lupus erythematosus, gouty tophi, } \\
\text { extragenital (cutaneous) lichen sclerosus, lupus vulgaris, lymphomatoid papulosis, } \\
\text { telangiectasia macularis eruptiva perstans, morphea, phthiriasis }\end{array}$ \\
\hline 2010 & 11 & $\begin{array}{l}\text { Diffuse cutaneous reticulohistiocytosis, sarcoidosis, Bullous scabies, crusted } \\
\text { (Norwegian) scabies, vasculitis (leukocytoclastic vasculitis), white fibrous papulosis } \\
\text { of the neck, demodicidosis, pagetoid reticulosis (Woringer-Kolopp type), pitted } \\
\text { keratolysis, pityriasis lichenoides et varioliformis acuta (PLEVA), tick bite }\end{array}$ \\
\hline 2011 & 9 & $\begin{array}{l}\text { Self-healing Langerhans cell histiocytosis (single nodule), dermatitis, dyschromatosis } \\
\text { symmetrica hereditaria, granuloma faciale, keratosis follicularis squamosa, keratosis } \\
\text { pilaris, solitary mastocytoma, other clinical forms of cutaneous mastocytosis (i.e., } \\
\text { nodular and plaque-type mastocytosis), nevus depigmentosus }\end{array}$ \\
\hline
\end{tabular}


Table 1 continued

Year Number of newly described Dermatoses dermatoses

201211

Poison ivy (Toxicodendron) dermatitis (black-spot poison ivy dermatitis), pseudolymphomatous folliculitis, follicular psoriasis, trichobacteriosis (trichomycosis) axillaris, acne, lichen amyloidosis, macular amyloidosis, aquagenic keratoderma, friction melanosis, Grover disease (Darier-like subtype), lichen simplex chronicus

Pseudoxanthoma elasticum-like papillary dermal elastolysis, papulopustular rosacea, granulomatous rosacea, milia-like idiopathic calcinosis cutis, delusions of parasitosis, granuloma annulare, genital lichen sclerosus, classic patch-type mycosis fungoides, perforating folliculitis, pityriasis rubra pilaris (classic form), porokeratosis ptychotropica, localized argyria

Inverse psoriasis, Riehl melanosis, Erythematotelangiectatic rosacea, Glandular rosacea, Phymatous rosacea, Trombiculiasis, Wasp (hymenoptera, vespidae) stings, Confluent and reticulated papillomatosis of Gougerot-Carteaud, Cutaneous collagenous (long-standing lesions), Seborrheic dermatitis, Digital myxoid pseudocyst, Digitate hyperkeratosis, Dyskeratosis congenita, Lichen planus pigmentosus, Lupus miliaris disseminatus faciei, Miliaria rubra, Mycetoma, Hyperpigmented mycosis fungoides, Poikilodermatous mycosis fungoides, Pediculosis corporis

Pretibial myxedema, Prurigo nodularis, Pseudofolliculitis corporis, Guttate psoriasis, Terra firma-forme dermatosis, Systemic amyloidosis (periocular papules), BRAFinhibitor induced acantholytic dyskeratosis, Cutaneous blastomycosis, Dermanyssus gallinae mite cutaneous infestation, Dermatomyositis (Gottron's sign), Dermatosis neglecta, Hypertrophic discoid lupus erythematosus, Chronic GVHD, Idiopathic guttate hypomelanosis, Lichen nitidus, Actinic lichen planus, Acute generalised lichen planus, Annular atrophic lichen planus, Follicular lichen planus, Genital annular lichen planus, Hypertrophic lichen planus, Hypopigmented mycosis fungoides, Elastosis perforans serpiginosa, Reactive perforating collagenosis, Granulomatous purpuric pigmentary dermatosis, Pityriasis lichenoides chronica, Circumscribed juvenile pityriasis rubra pilaris, Pityriasis versicolor, Pseudoxanthoma elasticum 
Table 1 continued

Year Number of newly described Dermatoses dermatoses

$2016 \quad 42$

$2017 \quad 35$
Achromic pityriasis versicolor, Plasma cell balanitis, Poikiloderma vasculare atrophicans, Progressive macular hypomelanosis, Pruritic folliculitis of pregnancy, Reticulate acropigmentation of Kitamura, Rheumatoid nodules, Subacute cutaneous lupus erythematosus, Condylomata lata, Thaumetopoea pityocampa cutaneous reactions, Tinea manuum, Tinea of vellus hair, White Piedra, Acute generalized exanthematous pustulosis (AGEP), Primary cutaneous nodular amyloidosis, Bed bug (Cimex lectularius) bites, Berloque dermatitis, Erythema ab igne, Erythrosis pigmentosa peribuccalis, Exanthematous (morbilliform) drug eruption, Pseudomonas folliculitis, Generalized essential telangiectasia, Acute GVHD, Hyperkeratosis lenticularis perstans, Jellyfish stings, Lymphocytoma cutis, Marginal zone B-cell lymphoma, Follicle center B-cell lymphoma, Diffuse large B-cell lymphoma, Schamberg disease, Majocchi disease, Linear purpuric pigmented dermatosis, Post-inflammatory hypopigmentation, Pseudoxanthoma elasticum (perforating variant), Contagious ecthyma (ORF), Kyrle disease, Pityriasis rubra pilaris (Erythrodermic), Tinea incognito, Staphylococcal scalded skin syndrome, Toxic epidermal necrolysis, Pseudoangiomatous xanthelasmoid mastocytosis, Cutaneous collagenous vasculopathy (recent lesions), Grover disease (spongiotic subtype)

Langerhans cell histiocytosis, Generalized argyria, Eruptive pseudoangiomatosis, Acral persistent papular mucinosis, Acral pseudolymphomatous angiokeratoma of children, Pretibial pruritic papular dermatitis, Generalized eruptive histiocytosis, Disseminated cryptococcosis with cutaneous involvement, Chromoblastomycosis, Focal dermal hypoplasia, Peruvian wart, Ashy dermatosis, Milker's nodule, Pachydermodactyly, Borderline tuberculoid leprosy, Lane's Disease (Erythema Palmare Hereditarium), Hailey-Hailey disease, Acrokeratosis verruciformis of Hopf, Perforating lichen nitidus, Erythromelanosis Follicularis Faciei et Colli, Syphilis (palmar syphiloderm), Histoid leprosy, Unilateral nevoid telangiectasia, Multicentric reticulohistiocytosis, Livedoid vasculopathy, Kikuchi's disease, Acute cutaneous leishmaniasis, Seborrheic Melanosis, Wound myiasis, Foreign body, Eruptive xanthomas, Primary cutaneous CD 30+ anaplastic large cell lymphoma, Folliculotropic mycosis fungoides, Ichthyosis vulgaris, Follicular porokeratosis 
Table 1 continued

Year Number of newly described Dermatoses dermatoses

$2018 \quad 27$

Pityriasis alba, necrobiotic xanthogranuloma, papuloerythroderma of Ofuji, pityrosporum folliculitis, idiopathic eruptive macular pigmentation, ash-leaf macules, amyloidosis cutis dyschromica, annular elastolytic giant cell granuloma, sporotrichosis, recurrent cutaneous eosinophilic vasculitis, methotrexate-associated lymphoproliferative disorder, tinea manuum, colloid milium, acral speckled hypomelanosis, pityriasis rotunda, idiopathic follicular mucinosis, disseminated strongyloidiasis, pseudo-Kaposi sarcoma, anetoderma, cutaneous loxoscelism, acrokeratoelastoidosis, post-kala-azar dermal leishmaniasis, Bier spots, poikiloderma of Civatte, pseudolymphoma, idiopathic facial aseptic granuloma, candidal balanitis

31

Pemphigus vulgaris, necrotizing vasculitis, calciphylaxis, DRESS syndrome, Mycobacterium marinum skin infection, systemic lupus erythematosus (malar rash), prurigo pigmentosa, eruptive xanthomas, atrophoderma of Pasini and Pierini, cydnidae pigmentation, cutaneous focal mucinosis, miliary osteoma cutis, primary cutaneous $\mathrm{CD}^{+}$small/medium sized pleomorphic T cell lymphoproliferative disorder, primary cutaneous acral $\mathrm{CD} 8^{+} \mathrm{T}$ cell lymphoma, Behcet's disease, keratosis lichenoides chronica, scleromyxedema, clear cell papulosis, Majocchi's granuloma, endogenous ochronosis, infectious folliculitis (fungal, bacterial, parasitic, and viral), eosinophilic folliculitis, palisaded neutrophilic and granulomatous dermatitis, Talaromyces (Penicillium) marneffei infection, tuberculoid leprosy, borderline lepromatous leprosy, lepromatous leprosy, type 1 lepra reaction, type 2 lepra reaction, cutaneous intravascular large B cell lymphoma, pustular psoriasis

Tuberculosis verrucosa cutis, lichen scrofulosorum, focal epithelial hyperplasia, pigmented purpuric lichenoid dermatitis of Gougerot and Blum, chronic superficial scaly dermatitis (small-plaque parapsoriasis), chilblain-COVID-19-like skin lesions, follicular Dowling-Degos disease, acanthosis nigricans, senile gluteal dermatosis, annular epidermolytic ichthyosis, childhood granulomatous periorificial dermatitis, incontinentia pigmenti, Kindler syndrome, syphilis (penile annular syphiloderm), necrolytic acral erythema, granulosis rubra nasi, lipoid proteinosis

\section{ACKNOWLEDGEMENTS}

Funding. No funding or sponsorship was received for this study or publication of this article.
Authorship. All named authors meet the International Committee of Medical Journal Editors (ICMJE) criteria for authorship for this article, take responsibility for the integrity of the work as a whole, and have given their approval for this version to be published. 
Author contributions. EE: concept, design, and writing of the manuscript. The author read and approved the final manuscript.

Disclosures. Enzo Errichetti has nothing to disclose.

Compliance with Ethics Guidelines. This article is based on previously conducted studies and does not contain any new studies with human participants or animals performed by the author.

Open Access. This article is licensed under a Creative Commons Attribution-NonCommercial 4.0 International License, which permits any non-commercial use, sharing, adaptation, distribution and reproduction in any medium or format, as long as you give appropriate credit to the original author(s) and the source, provide a link to the Creative Commons licence, and indicate if changes were made. The images or other third party material in this article are included in the article's Creative Commons licence, unless indicated otherwise in a credit line to the material. If material is not included in the article's Creative Commons licence and your intended use is not permitted by statutory regulation or exceeds the permitted use, you will need to obtain permission directly from the copyright holder. To view a copy of this licence, visit http:// creativecommons.org/licenses/by-nc/4.0/.

\section{REFERENCES}

1. Errichetti E, Zalaudek I, Kittler H, et al. Standardization of dermoscopic terminology and basic dermoscopic parameters to evaluate in general dermatology (non-neoplastic dermatoses): an expert consensus on behalf of the International Dermoscopy Society. Br J Dermatol. 2020;182:454-67.

2. Errichetti E. Dermoscopy of inflammatory dermatoses (inflammoscopy): an up-to-date overview. Dermatol Pract Concept. 2019;9:169-80.

3. Errichetti E, Stinco G. Dermoscopy in general dermatology: a practical overview. Dermatol Ther (Heidelb). 2016;6:471-507.

4. Lallas A, Giacomel J, Argenziano G, et al. Dermoscopy in general dermatology: practical tips for the clinician. Br J Dermatol. 2014;170:514-26.

5. Vos MHE, Nguyen KP, Van Erp PEJ, Van de Kerkhof PCM, Driessen RJB, Peppelman M. The value of (video)dermoscopy in the diagnosis and monitoring of common inflammatory skin diseases: a systematic review. Eur J Dermatol. 2018;28:575-96.

6. Errichetti E, Stinco G. The practical usefulness of dermoscopy in general dermatology. G Ital Dermatol Venereol. 2015;150:533-46.

7. Argenziano G, Fabbrocini G, Dfino M. Epiluminescence microscopy. A new approach to in vivo detection of Sarcoptes scabiei. Arch Dermatol. 1997;133: 751-3.

8. Elsner E, Thewes M, Worret WI. Cutaneous larva migrans detected by epiluminescent microscopy. Acta Derm Venereol. 1997;77:487-8.

9. Vázquez-López F, Kreusch J, Marghoob AA. Dermoscopic semiology: further insights into vascular features by screening a large spectrum of nontumoral skin lesions. Br J Dermatol. 2004;150:226-31. 